\title{
P-cadherin expression in glandular lesions of the uterine cervix detected by liquid-based cytology
}

\author{
A. Longatto Filho ${ }^{\star \dagger}$, A. Albergaria ${ }^{\ddagger}$, J. Paredes ${ }^{\ddagger}$, M. A. R. Moreira ${ }^{\S}$, F. Milanezi ${ }^{\dagger \neq}$ \\ and F. C. Schmitt ${ }^{\dagger, t}$ - \\ *Pathology Division of Adolfo Lutz Institute, São Paulo, SP, Brazil, `School of Health Sciences, University of Minho, Braga, \\ Portugal, "Institute of Molecular Pathology and Immunology of the University of Porto (IPATIMUP), Porto, Portugal, \\ ${ }^{\S}$ Departments of Pathology, Imageology, and Gynaecology of the Medical School of the Federal University of Goiás, Goiânia, \\ Goiás, Brazil and ${ }^{\top}$ Medical School of Porto, University of Porto, Porto, Portugal
}

Accepted for publication 1 November 2004

A. Longatto Filho, A. Albergaria, J. Paredes, M. A. R. Moreira, F. Milanezi and F. C. Schmitt

\section{P-cadherin expression in glandular lesions of the uterine cervix detected by liquid-based cytology}

Objective: To study P-cadherin aberrant expression as a possible marker for cervical adenocarcinomas in cytological samples.

Methods: We studied P-cadherin immunoexpression in liquid-based cervical cytology samples of biopsy-proven cervical lesions.

Results: We found a statistically significant correlation between P-cadherin expression and a cytological diagnosis of malignancy, either glandular or squamous $(P<0.0001)$. Twenty-two of 33 malignant cases showed $\mathrm{P}$-cadherin membrane staining. None of the 30 benign cases tested showed membrane staining, but three of them displayed an aberrant nuclear P-cadherin expression.

Conclusions: We concluded that P-cadherin can be used to discriminate between malignant and benign cervical cytological specimens, but not to discriminate glandular from squamous lesions.

Keywords: P-cadherin, uterine cervix, glandular lesions, liquid-based cytology

\section{Introduction}

Cervical adenocarcinoma is increasing in incidence worldwide, as well as in the relative proportion between this lesion and squamous cell carcinoma, particularly among young women. ${ }^{1-4}$ However, in contrast to screening for squamous lesions of the cervix, cervical smears have not been shown to reduce the incidence of adenocarcinoma. ${ }^{5-8}$ Difficulties in the interpretation of cytological findings may be responsible for both overdiagnosis and underdiagnosis of glandular lesions. For example, the diagnosis of atypical glandular cells of undetermined significance

Correspondence:

Fernando C. Schmitt, MD, PhD, MIAC, IPATIMUP, Rua Roberto Frias s/n, 4200 Porto, Portugal

Tel.: 35122557 0700; Fax: 35122557 0799;

E-mail: fschmitt@ipatimup.pt
(AGUS) is associated with a wide range of significant pathologies, many of them being considered squamous lesions. ${ }^{9,10}$ Patients with AGUS are usually referred for colposcopic examination and other procedures, such as endometrial aspiration, endocervical curettage, as well as cervical conization. Based on this fact, pathologists are required to report not only whether the smears favour neoplastic changes, but also the origin of the abnormal cells. ${ }^{1,12}$

Liquid-based cervical cytology (LBC) does offer some improvement in the detection rate of glandular abnormalities; moreover, molecular markers are also useful in distinguishing cervical adenocarcinoma from benign mimics. ${ }^{4,13}$ Immunohistochemical staining of MIB-1, bcl-2, CEA and p53 have shown potential in discriminating between cervical glandular abnormalities. ${ }^{14-18}$ Recently, the use of p16 ${ }^{\text {INK4a }}$ immunocytochemical reaction has emerged as an adjunct tool to distinguish cellular alterations that predict high-grade 
lesions, ${ }^{4,13}$ alone or associated with Ki-67, a cell cycle marker. ${ }^{14}$ pl6 protein is a cyclin-dependent kinase inhibitor, the increased levels of which appear to be related to high-risk HPV infection. Therefore, its overexpression is believed to address the nature of lesions of undetermined significance. ${ }^{4,11,13}$ Nieh et al. ${ }^{13}$ studied the $\mathrm{pl}^{\mathrm{INK} 4 \mathrm{a}}$ expression in cervical smears with AGUS and found that it was a highly sensitive and specific marker for neoplasia. Additionally, Akpolat et al. ${ }^{14}$ have also found an association between the expression of $\mathrm{pl}^{\mathrm{INK} 4 \mathrm{a}}$ and Ki-67 to identify high-grade and malignant lesions.

Recently, P-cadherin expression has been thought to be a useful tumour prognostic marker in some situations. ${ }^{18-23}$ P-cadherin-related tumour progression was observed in ovarian cancer, ${ }^{19}$ high-grade breast carcinoma ${ }^{20,21}$ and uterine cervix intraepithelial lesions. ${ }^{18,22}$

P-cadherin belongs to a superfamily of calciumdependent cell-cell adhesion glycoproteins expressed on the cell surface membrane. ${ }^{24}$ These molecules are thought to participate in several pathological conditions, such as cancer, and in some biological processes, including embryo development. ${ }^{25}$ Cadherins have also been implicated in a number of signalling pathways that regulate cellular activities, and it seems that the information received from cell-cell signalling, cell-matrix signalling, and growth factor signalling determines ultimate cellular phenotype and behaviour of normal or malignant cells. $^{24-26}$

More interestingly, P-cadherin aberrant expression was reported as a possible marker for glandular invasive malignancy of the uterine cervix. ${ }^{18}$ The authors studied archival paraffin block samples and showed that P-cadherin, normally confined to basal epithelial cells and not observed in benign glands, was aberrantly expressed in $96 \%$ (23 of 24 cases) of invasive adenocarcinomas and in $25 \%$ (two of eight cases) of adenocarcinoma in situ.

Therefore, the use of this molecular marker in the discrimination between malignant and benign glandular lesions encouraged us to study P-cadherin expression in cervical LBC. To achieve this goal, we selected cytological samples of in situ and invasive biopsyproven adenocarcinoma, intraepithelial and invasive squamous lesions, and negative cases with normal squamous and glandular cells, in order to determine whether P-cadherin could be used as an adjunct tool in assessing glandular atypias. To the best of our knowledge this is the first report focusing on
P-cadherin expression in uterine glandular lesions assessed by LBC.

\section{Materials and methods}

\section{Cytology collection}

Archival cytological samples were retrieved from the files of the Department of Pathology of the Federal University of Goiás, Brazil. The samples had been previously collected at Araújo Jorge Hospital, Goiás, Brazil, during the year 2002, and were selected according to the following criteria: cases with a biopsy-proven cytological diagnosis of adenocarcinoma and related HPV-induced lesions (squamous intraepithelial lesions, adenosquamous carcinoma); and cases where normal glandular cells were numerous, with no evidence of uterine disease to use as control cases. The cytological diagnoses were reported using the 2001 Bethesda System. ${ }^{27}$

\section{$L B C$ preparation}

The cervical specimens for LBC (DNA-CITOLIQ ${ }^{\circledR}$ System) were collected with a cervical brush and placed in a tube containing $1 \mathrm{ml}$ of Universal Collection Medium ${ }^{\circledR}$ (UCM) (Digene Co., Gaithersburg, MD, USA). The DNA-CITOLIQ ${ }^{\circledR}$ System consists of an aluminium instrument, the PREPGENE, which holds a slide holder (LAMIGENE) and a polycarbonate filter holder (FILTROGENE), with $25 \mathrm{~mm}$ diameter membranes $(5 \mu \mathrm{m}$ pore size). Twelve slides are prepared simultaneously. Briefly, the tube is vortexed for 15 seconds, individually, at high speed, and $200 \mu \mathrm{l}$ of the UCM specimen is put onto the polycarbonate membrane, spreading the liquid uniformly on the entire membrane surface. Then, the PREPGENE lid is locked for 10 seconds. During this step, an imprint of the cells is carried from the membrane to the glass slide.

\section{Immunocytochemistry protocol}

All cases were manually processed according to the streptavidin-biotin-peroxidase technique and using a commercial detection system (Ultra Vision Detection System; Lab Vision Corporation, Fremont, CA, USA), following the manufacturer's instructions. Cytological specimens, previously stained by the Papanicolaou technique, were hydrated and heat-induced antigen retrieval was done using EDTA solution, $\mathrm{pH}$ 8.0 (Lab Vision Corporation) in a $98^{\circ} \mathrm{C}$ water-bath, for 
20 minutes. After cooling at room temperature for 20 minutes, endogenous peroxidase activity was blocked by treating the slides with $3 \%$ peroxide/ methanol solution for 10 minutes. Non-specific staining was eliminated by incubation with normal serum (UltraVison Block; Lab Vision Corporation) for 20 minutes. The primary monoclonal antibody to the extracellular domain of P-cadherin (clone 56; BD- Transduction Laboratories, Lexington, KY, USA), diluted 1:50, was applied to the slides and incubated overnight at room temperature in a humid chamber. After washing with PBS-Tween, the slides were incubated with a biotin-labelled secondary antibody followed by streptavidin-biotincomplex also from Lab Vision. The samples were then stained with a solution of 3,3-diaminobenzidine tetrahydrochloride (DAB) (Lab Vision Corporation), counterstained with Mayer's haematoxylin (Merck, Darmstadt, Germany), dehydrated and mounted.

Formalin-fixed, paraffin-embedded positive and negative controls were included in each run. Normal human skin tissue samples were used as positive control for P-cadherin expression (continuous membrane staining of the proliferative/basal cell layer).

\section{P-cadherin immunocytochemical evaluation}

The expression of P-cadherin was evaluated by light microscopy as positive or negative, giving particular attention to the cellular localization of the positive reaction. Positive immunocytochemical reaction was defined as a well-decorated, brownish membrane staining, with a faint residual cytoplasmic staining.

\section{Predictive value of $P$-cadherin expression}

The predictive negative and positive values of $\mathrm{P}$-cadherin positive reaction was calculated using the following formulas: NPV $=$ True negative $(\mathrm{TN}) / \mathrm{TN}+$ false negative $\times 100 \%$ and $\mathrm{PPV}=$ true positive $(\mathrm{TP})=\mathrm{TP}+$ false positive $\times 100 \%$, or $22 / 22=100 \%$.

\section{HPV status}

The HPV status of the cases had been previously evaluated using the Hybrid Capture II and PCR methodology. The results were correlated with P-cadherin immunoreaction.

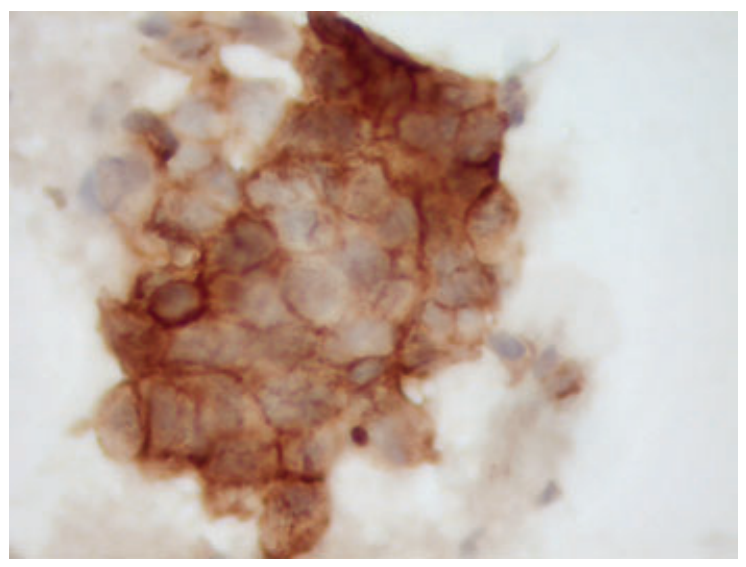

Figure 1. P-cadherin expression in a cervical adenocarcinoma cytological specimen. Note the intense membrane staining in the neoplastic cells (streptavidin-biotin-peroxidase, $\times 400)$.

\section{Results}

LBC slides of 33 biopsy-proven cases were selected for $\mathrm{P}$-cadherin immunocytochemical reactions. The positive immunoreactions were clearly demonstrated in clusters of squamous and/or glandular atypical cells (Figure 1). The overall distribution of P-cadherin reactions in positive cytology is depicted in Table 1.

The cases were classified as follows: one low-grade squamous intraepithelial lesion (LSIL), one atypical squamous lesion (ASC-H), seven high-grade squamous intraepithelial lesions (HSIL), two adenosquamous carcinoma, five squamous cell carcinomas (SCC), 13 adenocarcinomas in situ and four invasive adenocarcinomas. P-cadherin expression was observed in 22 of the $33(66.6 \%)$ analysed cases: nine squamous and 11 glandular lesions (Figure 1). Eleven cases (five squamous and six glandular lesions) did not show P-cadherin expression. The two cases of adenosquamous carcinoma were both positive for P-cadherin. Normal squamous and metaplastic cells were not stained by P-cadherin.

Thirty negative cases for malignancies and/or intraepithelial lesions were retrieved from the pathology files. All of them were negative for P-cadherin membrane staining; however three of 30 cases $(10 \%)$ showed a nuclear pattern of expression, and were considered as negative cases.

The correlation between P-cadherin expression and the diagnosis of a malignant lesion was statistically significant (Fisher's exact test: $P<0.0001$ ) (Table 2). 
Table 1. Distribution of P-cadherin expression and the positive cytological diagnoses

\begin{tabular}{|c|c|c|}
\hline Case & P-cadherin expression & Cytological diagnoses \\
\hline 1 & Negative & LSIL \\
\hline 2 & Negative & SCC \\
\hline 3 & Negative & HSIL \\
\hline 4 & Negative & HSIL \\
\hline 5 & Negative & Adenocarcinoma in situ \\
\hline 6 & Negative & Invasive adenocarcinoma \\
\hline 7 & Negative & Invasive adenocarcinoma \\
\hline 8 & Negative & Adenocarcinoma in situ \\
\hline 9 & Negative & HSIL \\
\hline 10 & Negative & Adenocarcinoma in situ \\
\hline 11 & Negative & Adenocarcinoma in situ \\
\hline 12 & Positive & HSIL \\
\hline 13 & Positive & Adenocarcinoma in situ \\
\hline 14 & Positive & HSIL \\
\hline 15 & Positive & Adenocarcinoma in situ \\
\hline 16 & Positive & Invasive adenocarcinoma \\
\hline 17 & Positive & Adenosquamous carcinoma \\
\hline 18 & Positive & Adenocarcinoma in situ \\
\hline 19 & Positive & SCC \\
\hline 20 & Positive & SCC \\
\hline 21 & Positive & ASC-H \\
\hline 22 & Positive & HSIL \\
\hline 23 & Positive & HSIL \\
\hline 24 & Positive & Adenocarcinoma in situ \\
\hline 25 & Positive & Invasive adenocarcinoma \\
\hline 26 & Positive & SCC \\
\hline 27 & Positive & Adenocarcinoma in situ \\
\hline 28 & Positive & Adenocarcinoma in situ \\
\hline 29 & Positive & Adenocarcinoma in situ \\
\hline 30 & Positive & SCC \\
\hline 31 & Positive & Adenocarcinoma in situ \\
\hline 32 & Positive & Adenosquamous carcinoma \\
\hline 33 & Positive & Adenocarcinoma in situ \\
\hline
\end{tabular}

LSIL, low-grade squamous intraepithelial lesion; HSIL, highgrade squamous intraepithelial lesion; SCC, squamous cells carcinoma; ASC-H, atypical squamous lesion favouring highgrade lesion.

Table 2. Correlation between P-cadherin (P-cad) expression and the samples cytological diagnoses

\begin{tabular}{lcll}
\hline & Benign & Malignant & Total \\
\hline P-cad negative & $30(100 \%)$ & $11(33.33 \%)$ & 41 \\
P-cad positive & $0(0.0 \%)$ & $22(66.67 \%)$ & 22 \\
Total & $30(100 \%)$ & $33(100 \%)$ & 63 \\
\hline
\end{tabular}

Fisher's exact test: $P<0.0001$.

The expression of P-cadherin was observed only in the specimens with a previous cytological diagnosis of malignant glandular or squamous lesion, while all the
Table 3. Correlation between P-cadherin (P-cad) expression and HPV status correlated with cytological diagnoses

\begin{tabular}{lcl}
\hline & HPV negative & HPV positive \\
\hline Negative cytology $(n=30)$ & $4(13.3 \%)$ \\
P-cad negative & $26(86.7 \%)$ & 0 \\
P-cad positive & 0 & $11(33.4 \%)$ \\
Positive cytology $(n=33)$ & 0 & $20(60.6 \%)$ \\
P-cad negative & $2(6.0 \%)$ & \\
P-cad positive &
\end{tabular}

cases previously diagnosed as benign did not express P-cadherin. The predictive negative and positive values of P-cadherin expression to detect malignancy were $73 \%(30 / 30+11 \times 100 \%)$ and $100 \%(22 / 22+$ $0 \times 100 \%)$.

From the 33 cases with HPV-related lesions, 31 $(94.0 \%)$ tested positive for oncogenic HPV with Hybrid Capture II methodology. The HPV status of the 30 negative control-cases tested with PCR methodology found four cases $(13.3 \%)$ were positive for oncogenic HPV (two HPV-16, one HPV-18 and one HPV-53). Table 3 summarizes these findings and also includes the correlation with P-cadherin expression.

\section{Discussion}

The diagnosis of adenocarcinoma in cytological material has become more common, mainly due to the increasing incidence of the disease, better endocervical sampling devices being used, and the morphological criteria for this diagnosis have been defined better and refined. ${ }^{5}$ However, there is still a significant variability in the interpretation of glandular lesions in cytology specimens. The use of additional HPV testing in atypias of glandular origin is particularly suggested for these cases. ${ }^{11,28}$ Efforts to improve the recognition of adenocarcinoma, primarily adenocarcinoma in situ, are a necessary caveat strategy to avoid incorrect categorization of such lesions in cervical smear examination. ${ }^{29,30}$

Recently, new parameters to evaluate cervical lesions have been studied. ${ }^{31-34}$ Accordingly, the preservative media of LBC systems are promising tools where immunocytochemical panels might be performed in order to optimize the cytological analysis. In particular pl6 is presumed to be a specific biomarker for high-risk HVP in women with cervical genital malignant and premalignant squamous lesions and is overexpressed in cervical adenocarcinoma (both in situ and invasive). ${ }^{31}$ Additionally, Ki-67 expression 
in intraepithelial lesions is a strong predictor of progression and seems to increase progressively in parallel with increasing aggressiveness of the lesions $^{32}$; besides that, it also appears correlated with p16 expression. ${ }^{33}$ Concomitant p53 and pl6 overexpression in patients with high-risk HPV infection is strongly associated with adenocarcinoma status. ${ }^{34}$

The premise of our study was to evaluate P-cadherin expression in LBC specimens, essentially to investigate the value of this molecular marker in cytological examination of cervical samples.

Aberrant P-cadherin is associated with a proliferative phenotype related to ulceration and neoplastic transformation, which may confer a survival advantage to these cells; but the relative functional role of $\mathrm{P}$-cadherin and the molecular mechanisms underlying $\mathrm{P}$-cadherin/catenin interactions have yet to be elucidated. ${ }^{35}$ As P-cadherin is known to be expressed in various tissues during embryogenesis, and also in the proliferative cells of normal epithelia, the expression of P-cadherin in carcinomas may be an oncofoetal phenomenon and/or may reflect its marked proliferative potential. ${ }^{36}$

The regulation of cadherin expression was previously reported in squamous intraepithelial lesions suggesting that the progression of such lesions was characterized by the gradual suppression of E-cadherin expression while P-cadherin became predominant. $^{22}$ Subsequently, P-cadherin evaluation was suggested for endocervical lesions as a potential marker of malignancy. ${ }^{18}$

We found a statistically significant correlation between P-cadherin expression and a previous cytological diagnosis of malignancy, either glandular or squamous, including intraepithelial squamous lesions. On the other hand, we observed an inverse correlation between the expression of this molecular marker and the benign samples (classified as negative for intraepithelial lesion or other malignancies by the 2001 Bethesda System ${ }^{27}$ ). P-cadherin can be used to discriminate between malignant and benign cervical cytological specimens, with predictive positive and negative values of $100 \%$ and $73 \%$, respectively. However, P-cadherin positivity does not allow discrimination between glandular and squamous lesions.

Likewise, negative status for oncogenic HPV infection strongly correlated with negative cytology and negative P-cadherin immunoreactivity $(86.7 \%)$. Conversely, positive HPV status correlated with positive cytology and positive P-cadherin immunoreactivity in $60.6 \%$.
We also found an unusual P-cadherin nuclear expression in normal endocervical cells in three of the 30 negative samples. This staining pattern has never been described for P-cadherin, in either benign cells or malignant lesions. Whether this staining pattern represents a real nuclear localization of P-cadherin or a cross-reaction with other nuclear factors, remains to be clarified. E-cadherin nuclear expression was observed by Han et al. ${ }^{37}$ in Merckel cell carcinomas; however, these authors, did not elucidate this unexpected finding also.

In conclusion, the improved morphological details of cervical glandular lesions provided by LBC, associated with an immunocytochemical search for the expression of molecular markers, such as P-cadherin, p16, p53 and Ki-67, could minimize the difficulties in interpretation of glandular and squamous lesions of undetermined significance.

\section{References}

1. Waggoner SE. Cervical cancer. Lancet 2003;361:2217-25.

2. Baalbergen A, Ewing-Grham PC, Hop WCJ, Struijk P, Helmerhorst TJM. Prognostic factors in adenocarcinoma of the uterine cervix. Gynecol Oncol 2004;92:262-7.

3. Smith HO, Tiffany MF, Qualls CR, Key CR. The rising incidence of adenocarcinoma relative to squamous cells carcinoma of the uterine cervix in the United States: a 24-year population-based study. Gynecol Oncol 2000;78:97-105.

4. Schorge JO, Lea JS, Elias KJ et al. P16 as a molecular biomarker of cervical adenocarcinoma. Am J Obstet Gynecol 2004; 190:668-73.

5. Renshaw AA, Mody DR, Lozano RL et al. Detection of adenocarcinoma in situ of the cervix in Papanicolaou tests. Comparison of diagnostic accuracy with other highgrade lesions. Arch Pathol Lab Med 2004;128:153-7.

6. Nanda K, McCrory DC, Myeres ER, Bastian LA, Hasselblad $\mathrm{V}$ et al. Accuracy of the Papanicolaou test in screening for and follow up of cervical cytologic abnormalities: a systematic review. Ann Intern Med 2000;132:810-9.

7. Moriaty AT, Wilbur D. Those gland problems in cervical cytology: faith or fact? Observations from the Bethesda 2001 terminology conference. Diagn Cytopathol 2003;28:171-4.

8. Segal A, Frost FA, Miranda A, Fletcher C, Sterrett GF. Predictive value of diagnoses of endocervical glandular abnormalities in cervical smears. Pathology 2003;35:198203.

9. Selvaggi S. Cytologic features of high-grade squamous intraepithelial lesions involving endocervical glands in ThinPrep ${ }^{\circledR}$ cytology. Diagn Cytopathol 2002;26:181-5. 
10. Wright VC. Cervical squamous and glandular intraepithelial neoplasia: identification and current management approaches. Salud Publica Mex 2003;45:S417-29.

11. Bekkers RLM, Bulten J, Wiersma-van Tilburg A et al. Coexisting high-grade glandular and squamous cervical lesions and human papillomavirus infections. BJM 2003;89:886-90.

12. Tam KF, Cheung ANY, Liu KL et al. A retrospective review on atypical glandular cells of undetermined significance (AGUS) using the Bethesda 2001 classification. Gynecol Oncol 2003;91:603-7.

13. Nieh S, Chen SF, Chu TY, Lai HC, Fu E. Expression of pl $6^{\text {INK4a }}$ in Pap smears containing atypical glandular cells from the uterine cervix. Acta Cytol 2004;48:173-80.

14. Akpolat I, Smith DA, Ramzy I, Chirala M, Mody DR. The utility of pl6INK4a and Ki-67 staining on cell blocks prepared from residual thin-layer cervicovaginal material. Cancer Cytopathol 2004;102:42-9.

15. Cameron RI, Maxwell P, Jenkins D, McCluggage WG. Immunohistochemical staining with MIB 1, bcl-2, and pl6 assists in the distinction of cervical glandular intraepithelial neoplasia from tubo-endometrial metaplasia, endometriosis and microglandular hyperplasia. Histopathology 2002;41:313-21.

16. Cina SJ, Richardson MS, Austin RM, Kurman RJ. Immunohistochemical staining for Ki-67 antigen, carcinoembryonic antigen, and p53 in the differential diagnosis of glandular lesions of the cervix. Mod Pathol 1997; 10:176-80.

17. Zarka TA, Han AC, Edelson MI, Rosenblum NG. Expression of cadherins, p53, and BCL2 in small cell carcinoma of the cervix: potential tumor suppressor role for N-cadherin. Int J Gynecol Cancer 2003; 13:240-43.

18. Han AC, Edelson MI, Soler AP et al. Cadherin expression in glandular tumors of the cervix. Aberrant P-cadherin as a possible marker of malignancy. Cancer 2000;89:2053-8.

19. Patel IS, Madan P, Getsios S, Bertrand MA, MacCaman $\mathrm{CD}$. Cadherin switching in ovarian cancer progression. Int $J$ Cancer 2003; 106:172-77.

20. Paredes J, Milanezi F, Viegas L, Amendoeira I, Schmitt F. P-cadherin expression is associated with high-grade ductal carcinoma in situ of the breast. Virchows Arch 2002;440:16-21.

21. Paredes J, Milanezi F, Reis-Filho JS et al. Aberrant P-cadherin expression: is it associated with estrogenindependent growth in breast cancer? Pathol Res Pract 2002; 198:795-801.

22. Boer CJ, van Dorst E, van Krieken $\mathrm{H}$ et al. Changing roles of cadherins and catenins during progression of squamous intraepithelial lesions in uterine cervix. Am J Pathol 1999;155:505-15.
23. Chen GTC, Tai CT, Yen LS, Yang TC, Tsai HD. Identification of the cadherin subtypes present in the human peritoneum and endometriotic lesions: potential role for P-cadherin in the development of endometriosis. Mol Reprod Dev 2002;62:289-94.

24. Vleminckx K, Kemler R. Cadherins and tissue formation. Bio Essays 1999;21:211-20.

25. Wheelock MJ, Johnson KR. Cadherins as modulators of cellular phenotype. Annual Review of Cell and Dev Biol 2003; 19:207-35.

26. Zigmond S. Formin' adherens junctions. Nature Cell Biol 2004;6:12-14.

27. Solomon D, Davey D, Kurman R et al. The 2001 Bethesda System. Terminology for reporting results of cervical cytology. JAMA 2002;287:2114-9.

28. Krane JF, Lee KR, Sun D, Yuan L, Crum CP. Atypical glandular cells of undetermined significance. Am J Clin Pathol 2004;121:87-92.

29. Oliveira ERZM, Derchain SFM, Rabelo-Santos SH et al. Detection of high-risk human papillomavirus (HPV) DNA by hybrid capture II in women referred due to atypical glandular cells in the primary screening. Diagn Cytopathol 2004;31:19-22.

30. Nonogaki S, Wakamatsu A, Longatto Filho A et al. Hybrid Capture II and polymerase chain reaction for identifying HPV infections in samples collected in a new collection medium: a comparison. Acta Cytol 2004;48:514-20.

31. Klaes R, Friedrich T, Spitkovsky D et al. Overexpression of pl $16^{\text {INK4A }}$ as a specific marker for dysplastic and neoplastic epithelial cells of the cervix. Int J Cancer 2001;92:276-84.

32. Maeda MYS, Simões M, Wakamatsu A et al. Relevance of the rates of PCNA, Ki-67 and p53 expression according to the epithelial compartment in cervical lesions. Pathologica, 2001;93:189-95.

33. de Putte GV, Kristensen GB, Lie K, Baeklandt M, Holm R. Cyclins and proliferation markers in early squamous cervical carcinoma. Gynecol Oncol 2004;92:40-6.

34. Zielinski GD, Snijders PJF, Rozendaal L et al. The presence of high-risk HPV combined with specific p53 and pl6INK4A expression patterns points to high-risk HPV as the main causative agent for adenocarcinoma in situ and adenocarcinoma of the cervix. J Pathol 2003;201:535-43.

35. Sanders DS, Perry I, Hardy R, Jankowski J. Aberrant $\mathrm{P}$-cadherin expression is a feature of clonal expansion in the gastrointestinal tract associated with repair and neoplasia. J Pathol 2000;190:526-30.

36. Shimoyama T, Hirohashi S. Expression of E- and P-cadherin in gastric carcinoma. Cancer Res 1991;51:2185-92.

37. Han AC, Soler AP, Tang CK, Knudsen KA, Salazar H. Nuclear localization of E-cadherin expression in Merkel cell carcinoma. Arch Pathol Lab Med 2000;124:1147-51. 